\title{
Cadres de pratiques et circulation des connaissances chez les potières de l' Arewa (Niger)
}

\section{Claire Corniquet}

\section{(2) OpenEdition}

1 Journals

Édition électronique

URL : https://journals.openedition.org/etudesafricaines/16597

DOI : 10.4000/etudesafricaines. 16597

ISSN : $1777-5353$

Éditeur

Éditions de l'EHESS

\section{Édition imprimée}

Date de publication : 30 mars 2011

Pagination : 87-114

ISBN : 978-2-7132-2297-9

ISSN : 0008-0055

\section{Référence électronique}

Claire Corniquet, "Cadres de pratiques et circulation des connaissances chez les potières de l' Arewa (Niger) », Cahiers d'études africaines [En ligne], 201 | 2011, mis en ligne le 05 mai 2013, consulté le 22 avril 2022. URL : http://journals.openedition.org/etudesafricaines/16597 ; DOI : https://doi.org/ 10.4000/etudesafricaines.16597

Ce document a été généré automatiquement le 22 avril 2022.

(c) Cahiers d'Études africaines 


\title{
Cadres de pratiques et circulation des connaissances chez les potières de l' Arewa (Niger)
}

\author{
Claire Corniquet
}

1 Le propre de l' archéologie est de saisir et de reconstituer l' histoire humaine au travers des productions matérielles. À partir $\mathrm{d}^{\prime}$ artefacts - dont la céramique constitue un corpus important - des regroupements stylistiques et morphologiques sont réalisés, qui servent à définir des "aires culturelles» géographiques et temporelles. Pour appréhender les modes de vie, les dynamiques sociales et culturelles des sociétés anciennes, l' accent est mis sur le contexte de consommation des objets. Bien que l' intérêt de telles analyses ne doive pas être remis en cause, il reste que les archéologues sont forcément limités par l' absence $\mathrm{d}$ ' accès direct aux savoirs pratiques de "ceux " dont ils examinent la culture matérielle. Or, $s^{\prime}$ ils influencent aussi les processus de distribution des artefacts et des techniques, les contextes de production sont longtemps restés absents des recherches archéologiques.

2 Ce manque se comble peu à peu grâce, notamment, aux études ethno-archéologiques. En Afrique, bon nombre de ces études concernent la chaîne opératoire de la poterie. Dans ce domaine, l' approche ethnographique a permis d' une part, de caractériser les techniques de fabrication et les contextes de production de la céramique et, d' autre part, de mettre en valeur la diversité des modalités pratiques et des nuances techniques identifiables au sein de différents groupes d' artisans. Ces études se sont attachées à situer $\mathrm{l}^{\prime}$ activité potière dans son contexte de pratique et ont de ce fait, dépassé l' analyse du simple artefact « céramique ».

3 La poterie, objet utilitaire encore courant sur l' ensemble du territoire africain, est un excellent média pour appréhender le rapport entre les logiques sociales et les logiques techniques. Cet artefact implique l' interaction d' une série d' acteurs (artisans, colporteurs, clients), tant dans la sphère de production que dans celle de la consommation. Cet aspect a été mis en valeur par des études consacrées aux pratiques céramiques actuelles, qui illustrent l' existence de liens forts entre les techniques 
utilisées par les artisans et leur identité sociale. Comme l' ont montré Mahias (2002) et Gosselain (2000), par exemple, une technique de façonnage ou une préparation de pâte peut être l' apanage d' un groupe linguistique, d' une caste ou d' un genre. Néanmoins, des différences dans les modes de préparation de pâte, de cuisson ou encore de décors sont fréquemment observées parmi des artisans se reconnaissant pourtant une même appartenance (quelle soit linguistique, de caste ou de genre). En d' autres termes, la pratique potière au sein de groupes $d$ 'individus ayant une même identité « linguistique » ou « statutaire » comporte autant de similitudes que de différences.

4 L' identité technique se construit constamment, dans le cadre de rapports sociaux ordinaires et au sein de contextes connus et pratiqués. L' approche historique de Berns (1989) tend à montrer également que la culture matérielle ne peut être abordée sans considérer le cadre historico-social dans lequel elle s' insère. Une culture matérielle commune ne permet pas toujours $d^{\prime}$ identifier un même groupe linguistique. Un examen plus minutieux montre que des appropriations ont lieu, suite à des contacts longs et répétés qui contribuent à donner du sens aux objets et aux techniques utilisées pour les façonner. La question du choix qu' effectue l' artisan à diverses étapes de la chaîne opératoire (Lemonnier 1993 ; Stark 1999) devient alors incontournable pour qui cherche à comprendre les pratiques actuelles autant que leur histoire. Ainsi, un groupe $\mathrm{d}^{\prime}$ individus peut partager une "palette de possible ", c' est-à-dire d' alternatives techniques, de connaissances mobilisables, qui fondent le "répertoire technique » $d^{\prime}$ une population et se constituent au fil du temps suite aux interactions entre les acteurs. En fonction du contexte social dans lequel se déroule l' activité, l' artisan peut choisir parmi ces éléments et moduler éventuellement celui-ci par la suite. Comme l' ont illustré Livingstone-Smith (2001) et Gosselain (2005), un artisan peut, au cours de sa carrière, adapter sa manière de faire en fonction de « normes changeantes » associées à son activité. Il ne s' agit pas forcément d' un changement vers un " mieux ", mais vers un " différent ", plus efficace socialement. Dans ce cas, le choix technique renvoie à une représentation modifiée $d^{\prime}$ une pratique adéquate. Il semble donc, $d$ ' une part, que le contexte donne du sens à la pratique et, $d^{\prime}$ autre part, que le choix technique est la réification $d^{\prime}$ une pratique jugée appropriée techniquement, socialement et économiquement.

Mais depuis les travaux entrepris par des pionniers comme d' Haudricourt, Mauss, Leroi Gourhan, et systématisés au sein de ce qu' on a coutume de désigner la «technologie culturelle» avec des personnalités comme Lemonnier, Sigaut, Cresswell, Guille-Escuret, ou Pfaffenberger, une question reste entière : sur quoi se portent les choix techniques? Comment décide-t-on d' adopter une autre manière de faire, une technique plutôt qu' une autre? Dans Technological Choices, Lemonnier (1993) postule que les techniques sont des productions sociales et que les choix s' opèrent dans $l^{\prime}$ interaction entre les acteurs et les systèmes techniques. Mais sous quelle forme et dans quel contexte prennent place ces interactions ? Et comment justifier le fait que le choix $\mathrm{d}$ ' une technique puisse concerner un groupe $\mathrm{d}^{\prime}$ individus tout entier?

6 À ce stade de la recherche, si nous voulons comprendre comment s' imbriquent le social et la technique, il est nécessaire d' identifier et de situer «les cadres de pratiques $»^{1}$ dans lesquels se déroule l' activité potière. La pratique céramique ne peut être séparée de la vie quotidienne de celles et ceux qui la mettent en œuvre. «Faire de la poterie » ne s' exerce pas dans une bulle temporelle et contextuelle particulière, ce $n^{\prime}$ est pas une pause dans l' espace-temps des individus. C' est une activité qui s' inscrit, au 
contraire, dans le quotidien des artisans et s' intègre entre la préparation d' un repas, une visite amicale, l' entretien de la concession, une attention donnée aux enfants, etc. Par ailleurs, si la pratique céramique $s^{\prime}$ exerce dans le contexte domestique, elle investit également l' espace villageois par le fait, notamment, que certaines étapes de la chaîne opératoire se déroulent hors de la concession. Lorsqu' une potière réalise un récipient, elle $\mathrm{n}^{\prime}$ est donc pas isolée, mais elle inscrit sa pratique dans un monde connu et habité. Sa technique est autant marquée par son apprentissage que par son identité «familiale », villageoise, linguistique ou encore par ses interactions avec des artisanes de son village ou d' ailleurs, ses amitiés ou ses inimitiés. Si l' on admet que toute pratique est située et que la situation donne du sens à la pratique (Suchman 1987; Brown et al. 1989; Visetti 1989; Wenger \& Lave 1991), alors, il devient impératif de mettre en évidence les situations de pratique autant que les cadres dans lesquels ces situations se développent. La situation fait référence au cadre physique dans lequel une activité prend place, aux actions qui la définissent, ainsi qu' aux personnes qu' elle met en scène. Aborder l' activité potière au travers des cadres de pratiques qui lui sont associés, permet notamment de mettre en évidence une série de relations qui échappent parfois au regard, soit parce qu' elles ne sont pas propres à la chaîne opératoire, soit parce que leur caractère périphérique tend à en minorer l' importance.

7 Partant de ce constat et sur la base $\mathrm{d}^{\prime}$ enquêtes menées auprès de potières de la région de l' Arewa (sud-ouest du Niger), nous mettrons en évidence les divers cadres de pratiques relatifs à la poterie et leur impact sur les « façons de faire » de cette région du Niger. Nous aborderons les "filières" par lesquelles circulent les connaissances au niveau local (villageois) et régional (intervillageois), afin d' approcher les mécanismes responsables des configurations techniques en présence dans la zone d' étude. Ainsi, et contrairement à la plupart des autres études sur la poterie, notre intérêt porte moins sur la chaîne opératoire stricto sensu que sur les « cadres » dans lesquels elle se déroule.

\section{Terrain de recherche : cadre géographique et historique}

Les données présentées dans cette étude sont issues d' enquêtes ethnographiques menées depuis 2004 au Niger ${ }^{2}$. Elles ont été recueillies auprès des potières de 36 localités situées dans trois régions administratives de l' Arewa (fig. 1): nord de la région de Dosso (départements de Dogondoutchi et Loga); sud-est de la région de Tilabéri (département de Filingue); sud-ouest de la région de Tahoua (départements de Birni N' konni et Illela). 


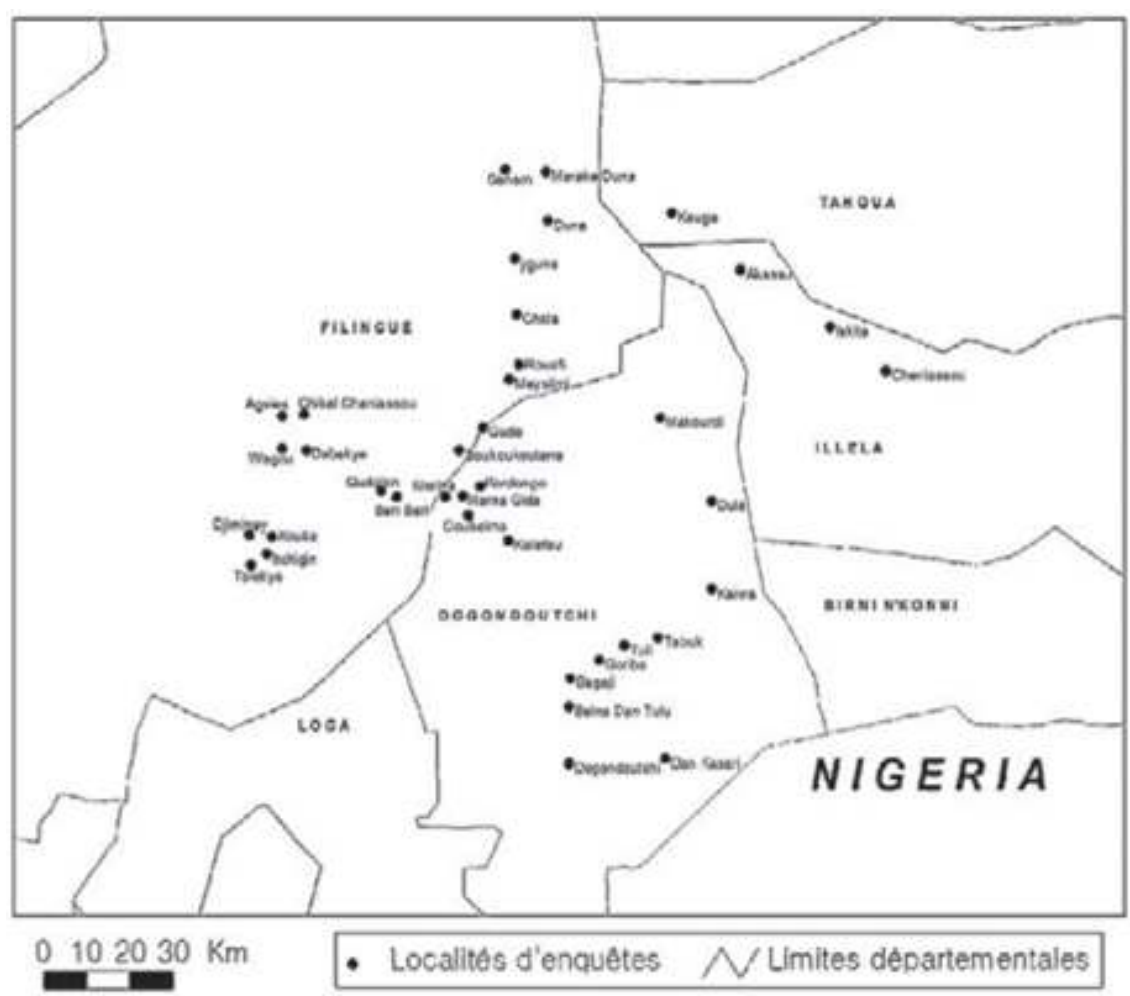

Fig. 1. - RéGion de l' Arewa et Villages dans lesquels nous aVons rencontré les artisanes

L' Arewa se situe au sud-ouest du Niger, dans la partie sahélienne du pays. Cette région est comprise entre Filingue, Dosso, Tahoua et Konni, quatre villes importantes du Niger méridional. Située en zone semi-aride parsemée d' arbustes et d' épineux, cette région se dessine comme un plateau coupé par les dallols (ou vallées) Bosso et Maouri, anciens affluents du fleuve Niger. Limitée à l' ouest par le Zarma Ganda et à l' est par l' Ader, l' Arewa septentrionale se trouve à la jonction des pays zarma/songhaï et hausa.

Bien que la zone d' étude soit essentiellement peuplée de locuteurs hausas ${ }^{3}, 1$ ' identité des habitants $n^{\prime}$ est pas monolithique. Nos interlocutrices sont toutes hausaphones, mais leur « appartenance » se décline sur une base régionale : suivant le lieu d' enquête, elles se présentent comme Hausa mawri, arewa, aderawa, bagube ou encore kurfawa et beri beri. De manière générale les Hausas présentent de grandes différences et il paraît plus adéquat de parler des peuples hausas katsinawa, gobirawa, arewa, aderawa, etc. L' origine en est si diverse qu' il est bien hasardeux de parler d' une " ethnie hausa » ou de "Hausa » au sens générique. Chacun de ces peuples a connu une expérience historique différente, qui s' est traduite par la création d' un royaume, d' une capitale propre et d' une ou plusieurs dynasties spécifiques (Hamani 1993 : 169). L' histoire du peuplement de l' Arewa illustre parfaitement la rencontre et la cohabitation de peuples d' origines différentes. Le terme « Arewa » désigne à la fois la zone septentrionale du dallol maouri et une des deux communautés "historiques " de cette région, située à l' extrémité occidentale de l' aire hausa (de Latour $1984: 273$ ). Ces deux communautés sont d' une part, les Gubawa ${ }^{4}$ (ou Bagube), qui s' affirment comme les premiers habitants de l' endroit, et, $d$ ' autre part, les Arewas ${ }^{5}$, guerriers venus du Bornu, qui se seraient imposés comme protecteurs à la population déjà présente. Au fil du temps, de petits groupes de populations se sont intégrés aux communautés arewas et c' est l' ensemble de ces couches de populations qui est désigné sous le terme de «maouri» (ibid.: 276-277). 
Cette appellation est la plus fréquemment utilisée au Niger, notamment par les Peuls et les Zarmas, pour désigner aussi bien le pays de Dogondoutchi que ses habitants. Mais les intéressés eux-mêmes emploient le terme Hausa d' Arewa pour la région et réservent ainsi l' appellation "Maouri " aux éléments superposés (Séré de Rivières 1965 : 49-50). Ainsi, il existe une certaine unité culturelle à l' échelle régionale - grâce notamment à la cohabitation de ces peuples d' origines diverses, qui se sont mélangés par le biais d' alliances matrimoniales - mais on ne peut parler d' « ethnie mawri » (de Latour 1984), car ce terme ne désigne qu' une région géographique dont les habitants s' identifient par leur nom de groupe.

\section{Communauté de potières et chaîne opératoire}

\section{Qu' est-ce qu' une communauté de potières ?}

11 Pourquoi parler de " communauté » en matière de production céramique dans la région de l' Arewa? À première vue, la poterie est une activité qui se pratique de manière individuelle: chaque potière ${ }^{6}$ maîtrise son ouvrage du début à la fin de la chaîne opératoire ; il n' y a pas d' atelier collectif, puisque chaque artisane possède un espace réservé au sein de la concession; le profit financier de la production est propre, puisque la totalité des gains, lors de la vente des récipients, revient à l' artisane. Néanmoins, les enquêtes de terrain révèlent qu' à chaque étape de la chaîne opératoire, $l^{\prime}$ artisane est en contact plus ou moins étroit avec $d^{\prime}$ autres praticiennes : apprenties, artisanes de sa localité ou d' autres localités. Si ces contacts sont parfois informels, des regroupements ont lieu régulièrement dans le cadre de certaines étapes de la chaîne opératoire. Mais bien que ceux-ci soient planifiés et engendrent des actions collectives, ils ne sont pas indispensables à la pratique de l' activité. En effet, une potière isolée est parfaitement capable de mener à bien toutes les étapes de son travail sans aide extérieure. Les regroupements semblent donc issus $d^{\prime}$ une volonté commune de collaborer et de s' entraider. Cette collaboration vise autant à alléger le travail par un partage des tâches, qu' à « être ensemble » par plaisir ou par habitude. Quatre cadres de pratiques peuvent être le théâtre d' une telle collaboration : l' atelier, le lieu de cuisson, le site d' extraction et le marché. Nous allons à présent en explorer les spécificités, en suivant le déroulement de la chaîne opératoire et en détaillant les opérations qui s' y déroulent.

\section{Chaîne opératoire et cadres de pratiques}

12 Le premier cadre de pratiques qui nous intéresse est le site d' extraction. $C^{\prime}$ est là que $l^{\prime}$ artisane se procure son argile, matière de base pour la réalisation $\mathrm{d}^{\prime}$ une céramique. Dans l' Arewa, les sites se situent généralement hors du village, dans un rayon de un à vingt kilomètres. Les artisanes s' y rendent à pied, en charrette ou en voiture. Deux sites sont généralement utilisés simultanément, situés à des distances et dans des environnements différents: une argile "blanche» est habituellement extraite à proximité du village (puits et collines) et une «noire» dans les mares ou les cours d' eau asséchés, à plus grande distance. $C^{\prime}$ est cette seconde catégorie de sites qui fait souvent l' objet $\mathrm{d}^{\prime}$ un partage entre artisanes de localités différentes, partage qui suscite des rencontres "extra villageoises " généralement fortuites. D' une manière générale, toutes les artisanes d' un même village fréquentent les mêmes sites d' 
extraction, y compris celles qui, originaires d' autres villages, avaient l' habitude de s' approvisionner ailleurs. En $d^{\prime}$ autres termes, les potières originaires $d^{\prime}$ autres villages adoptent généralement le ou les site(s) d' extraction utilisé(s) par les potières locales. L' extraction est une étape qui est rarement menée seule. En effet, la potière est fréquemment accompagnée d' enfants de sa concession et, dans certaines localités, les artisanes s' accordent pour aller chercher l' argile ensemble. Bien que l' acheminement jusqu' à la source puisse s' effectuer collectivement, la responsabilité du choix de l' argile est individuelle. En effet, chacune sélectionne un emplacement à chaque nouvelle saison de travail ${ }^{7}$, et y dégage la matière première. Il existe donc plusieurs zones d' extraction au sein $d$ ' un même site de prélèvement. Chaque potière a une conception personnelle des propriétés physiques que doit posséder l' argile, laquelle découle de son apprentissage et de sa pratique (Arnold 1972 : 38). Néanmoins, le site et la qualité de l' argile font souvent l' objet de discussions et de conceptions partagées entre celles fréquentant le même site: la qualité de l' argile est régulièrement considérée comme un élément distinctif entre deux productions villageoises: « $L^{\prime}$ argile $d^{\prime}$ ici, $c^{\prime}$ est mieux ", disent les potières de Kordongo ; " $C^{\prime}$ est pas pareil, l' argile est pas la même, $c^{\prime}$ est plus solide ", disent celles de Djiminey. Ces éléments sont évoqués fréquemment par les artisanes pour justifier le fait qu' elles ne fréquentent pas les mêmes sites d' extraction que leurs voisines proches ou pour distinguer leur production de celles qui exploitent des argiles différentes.

14 Le deuxième cadre de pratiques est $\mathrm{l}^{\prime}$ atelier, ou tout au moins l' espace réservé à la fabrication au sein de la concession ${ }^{8}$. Cet espace est occupé par la ou les potière(s) de la maisonnée, ainsi que les apprenties. Trois étapes de la chaîne opératoire s' y déroulent : la préparation de $\mathrm{l}^{\prime}$ argile ${ }^{9}$, le façonnage ${ }^{10}$ et la décoration des récipients. Lors de ces trois opérations, $\mathrm{l}^{\prime}$ artisane peut se faire assister d' une ou plusieurs apprentie(s). Il arrive également qu' elle travaille avec une autre potière de la concession ou d' un atelier voisin. Cette dernière est souvent un membre de la famille ou une amie. Ainsi, à Kordongo, Tukunti et Hadi partagent parfois le même atelier. Tukunti étant plus jeune, elle se rend dans l' atelier de Hadi et y fabrique ses récipients. Si l' une d' entre elles a terminé son ouvrage, elle prend parfois l' initiative de préparer la pâte de l' autre ou de $l^{\prime}$ aider dans le façonnage des récipients. Cet exemple n' est pas une exception, puisque dans ce village, un autre couple et un trio de potières opèrent de la même manière. Nous avons également retrouvé une situation similaire à Merina, un village situé à proximité de Kordongo. Néanmoins, « chacun a sa main " ${ }^{11}$, expliquent les potières, une expression régulièrement employée pour signifier que le travail de chacune est distinct: tant dans les faits que dans l' esprit des personnes interrogées, chaque production est singulière.

15 Le troisième cadre de pratiques est le site de cuisson. Réalisée en bordure du village, dans des dépressions ${ }^{12}$ prévues à cet effet, la cuisson s' effectue la plupart du temps avec l' aide d' apprenties et/ou d' autres potières du village, dans des emplacements que se partagent plusieurs potières. La création d' un site de cuisson est le fait de potières isolées, soit parce qu' elles sont les premières du village, soit parce qu' elles ne sont pas intégrées dans un réseau de connaissances leur permettant de partager un «trou » de cuisson, soit parce que des inimitiés les empêchent de partager le même trou, soit, encore, parce qu' elles ne sont pas du même « quartier » ou groupe social.

Bien que plusieurs personnes partagent un même trou, ce dernier « appartient » à celle qui l' a confectionné initialement ou qui en a hérité de sa mère. L' héritière responsable 
possède alors des droits et des obligations implicites quant à la gestion du site (par exemple le nettoyage de l' emplacement à chaque nouvelle saison de travail). Le groupe de cuisson constitué de potières et d' apprenties partageant un même trou ne varie pas, mais il se renouvelle néanmoins lorsqu' un membre cesse l' activité et que son apprentie directe ou une novice occupe sa place. Celles qui partagent le site sont donc des personnes dont le maître le fréquentait déjà, ou des artisanes intégrées dans le groupe via le réseau familial ou amical.

17 Les modalités de cuisson et les combustibles varient parfois au sein d' un même village. $C^{\prime}$ est le cas, notamment, à Kauge où quatre potières sont en activité : Aminatu, Dize, Bibata et Goma. Aminatu et Goma cuisent ensemble dans un trou distinct de celui de Dize et Bibata. Hormis Aminatu qui est de Kauge et qui y a effectué son apprentissage, les trois autres sont originaires $d^{\prime}$ autres villages où elles ont effectué leur formation. Elles expliquent leur association par le fait qu' elles $\mathrm{n}^{\prime}$ ont pas la même recette de préparation de pâte et $\mathrm{n}^{\prime}$ utilisent pas les mêmes combustibles lors de la cuisson. Dize et Bibata ajoutent à l' argile de la chamotte et de la balle de mil, alors qu' Aminatu et Goma ajoutent uniquement de la chamotte. Concernant la cuisson, toutes les quatre tapissent le trou d' une couche de tiges de mil avant d' y placer les récipients, mais Aminatu et Goma recouvrent alors ceux-ci de tiges de mil, tandis que Bibata et Dize les recouvrent de tiges de mil et de bouse de vache. À Kauge, les artisanes n' ont pas trouvé d' utilité, tant sociale que technique, pour homogénéiser leur "savoir-faire ». Elles ont, en effet, trouvé leur alter ego en matière de pratique.

D' une manière générale, les potières partageant le même site de cuisson - alors qu' elles ont parfois acquis leurs connaissances dans $d$ ' autres localités, avec des instructrices et selon des modalités différentes - tendent à développer une pratique commune. Lorsque la jeune potière arrive et ne trouve pas $\mathrm{d}$ ' " homologue technique ", elle s' adapte généralement à la pratique locale. À Kordongo, par exemple, Barrow a adapté sa préparation de pâte à celle du groupe de cuisson dans lequel elle s' est intégrée. L' exemple de Kauge est exceptionnel, car les modalités de cuisson sont généralement identiques pour l' ensemble des artisanes d' un même village, même si elles $\mathrm{n}^{\prime}$ en sont pas toutes originaires. Dans la majorité des cas, les différences de cuisson observées au sein $\mathrm{d}^{\prime}$ un même village ne concernent pas les combustibles utilisés, mais consistent en de petites variantes. Ainsi, à Kordongo, les membres de certains groupes de cuisson cassent les tiges de mil, alors que d' autres les gardent entières avant de les placer au-dessus des récipients. Bien qu' en apparence la technique soit identique, les " petites variantes » matérialisent une manière de faire, propre à chaque groupe de cuisson.

Enfin, le dernier cadre de pratiques est le marché, sur lequel l' essentiel de la production céramique est écoulée ${ }^{13}$. Ces marchés ont lieu à jours fixes et drainent des vendeurs et acheteurs dans un rayon de 20 à $30 \mathrm{~km}$. Il est courant que les potières fréquentent plusieurs marchés si ces derniers se trouvent à distance raisonnable. En règle générale, celui qui est le plus proche du village fait l' objet d' une fréquentation hebdomadaire. Les potières ne fréquentent $d$ ' autres points de vente que si elles en ont le temps où si elles $\mathrm{n}^{\prime}$ ont pas suffisamment vendu de récipients lors du marché régulier. La réputation et la taille du marché sont également des facteurs déterminants dans le choix des points de vente fréquentés par les potières. Néanmoins, les artisanes ne décident pas spontanément de se rendre sur un marché pour vendre leur production. Loin d' être aléatoire, la fréquentation d' un marché se transmet d' une 
génération à l' autre, dans une ou plusieurs localités. Les fabricantes y ont leurs habitudes et un emplacement " réservé ». Trois modes d' organisation sont observés à ce niveau : un regroupement par village d' artisanes d' origines différentes ; un partage de l' emplacement, quelle que soit l' origine ; un éclatement d' emplacements malgré une origine commune.

Les amitiés et inimitiés influencent le positionnement et la constance des personnes auprès desquelles les artisanes prennent place. Le marché apparaît comme un espace et un moment privilégié pour se retrouver et bavarder, mais également pour interagir avec celles qui viennent $d$ ' autres villages. Ces discussions peuvent avoir pour objet le savoir technique et les problèmes rencontrés, notamment à l' occasion de la préparation de la pâte ou de la cuisson. $C^{\prime}$ est également un moment où l' on peut prendre connaissance des goûts et des plaintes de la clientèle. Selon les personnes interrogées, ce type $d$ ' information peut ensuite être relayé auprès des potières du village qui n' en ont pas eu connaissance directement.

Nous sommes donc en présence d' une série de "cadres de pratiques » dans lesquels se développent diverses formes $\mathrm{d}$ ' interactions. Ces "cadres de pratiques » sont associés à quatre moments propices à la prise de connaissance des "façons de faire » et des «façons de voir » des autres potières du village, mais également de la clientèle et des changements qui permettraient éventuellement $l^{\prime}$ amélioration de la technique : $l^{\prime}$ extraction de l' argile, la cuisson, la vente sur le marché ${ }^{14} \mathrm{et}$, dans une moindre mesure, la pratique au sein de l' atelier. Ces quatre cadres correspondent à des espaces dans lesquels les artisanes d' une même localité participent à une "entreprise commune " (une cuisson collective par exemple) et sont engagées dans une pratique partagée avec des artisanes issues du même village ou d' autres localités (sur le site d' extraction ou au marché). L' analyse spatiale peut alors s' effectuer à deux niveaux: le niveau villageois (atelier et site de cuisson) et extra villageois (site d' extraction de l' argile et marché).

\section{Niveau villageois : fonctionnement de la communauté potière}

Les enquêtes ethnographiques effectuées dans la région de l' Arewa indiquent que les techniques de préparation de pâte, de façonnage ou encore de décoration et de cuisson sont, à quelques exceptions près, communes à l' ensemble des pratiquantes de l' entité villageoise, alors que celles-ci $n$ ' en sont pas forcément natives et ont parfois appris la poterie dans une autre localité. Une telle homogénéité s' expliquerait aisément si toutes les potières avaient appris dans le même village, avec la même « instructrice ». Or, certaines reconnaissent avoir modifié ou adapté leur technique - acquise, dans ce cas, pendant l' enfance dans un autre contexte villageois - en fonction des conventions en vigueur dans leur nouvel espace de pratique, leur village $d^{\prime}$ "accueil», habituellement celui de leur époux.

De façon générale, dans un village où se côtoient des potières qui ont acquis leurs connaissances dans d'autres localités, nos informatrices décrivent les spécificités de leur travail par un laconique "on fait tous pareil». Les changements technique et décoratif qui interviennent éventuellement au fil du temps sont effectués par l' ensemble des potières du village : «Faire tous pareil » prend la signification de «faire comme les autres ». Ceci paraît d' autant plus important que nos informatrices ont une 
connaissance relativement précise de la manière dont procède chaque artisane du village. On voit donc $d$ ' une part que les connaissances individuelles circulent $d$ ' une artisane à l' autre et, $d^{\prime}$ autre part, que les potières $d^{\prime}$ ' un même village constituent un groupe dont les membres se reconnaissent par le fait de «faire la même chose ».

Ainsi, ce groupe se développant à l' échelle de l' entité villageoise est assimilable à ce que Wenger et Lave (1991) appellent une communauté de pratique ${ }^{15}$. Les potières qui y " évoluent » techniquement s' inscrivent dans une histoire de pratiques partagées. Par le biais d' opérations collectives au cours desquelles elles s' entraident et échangent des informations, les artisanes constituent un répertoire partagé de connaissances et de savoir-faire, et développent un sentiment d' appartenance et d' engagement avec les autres pratiquantes. Ce processus s' ancre et se renforce dans le fait de faire « la même chose », ce qui entraîne une homogénéité progressive des pratiques.

\section{Fonctionnement d' une communauté de potières}

Le sentiment d' appartenance à une communauté (même informelle) et l' engagement qui s' établit entre les artisanes d' une même communauté ne se développent pas du jour au lendemain. Comme l' ont déjà illustré Wenger et Lave (1991), Wenger (1998), Chanal (2000) Geslin et Salembier (2002), Bowser et Paton (2008), c' est la pratique quotidienne d' une activité commune qui constitue le socle d' une communauté et qui en assure la pérennité. Lorsque l' on décortique la manière dont se déroule l' activité potière, il est clair que l' intégration et l' appartenance à un groupe de pratiquants se construisent et se négocient progressivement. La poterie, comme toute pratique, nécessite un apprentissage. La formation débute soit pendant l' enfance, soit après le mariage.

Lorsque l' apprentissage s' effectue pendant l' enfance, la novice s' engage d' abord dans des tâches mineures nécessitant peu de savoir technique et souvent considérées comme faciles, voire anodines ${ }^{16}$. À mesure que la novice acquiert de l' expérience, son activité devient plus centrale, en même temps qu' évoluent ses relations avec les autres membres. La novice s' engage dans un processus $d^{\prime}$ apprentissage, tout $d^{\prime}$ abord en périphérie de l' activité ${ }^{17}$. Dès le début de sa formation, l' apprentie est en contact avec $\mathrm{d}^{\prime}$ autres artisanes que son maitre, à l' occasion, notamment, $\mathrm{d}^{\prime}$ une cuisson, de $\mathrm{l}^{\prime}$ extraction de l' argile ou d' une vente sur un marché. Ussey, jeune apprentie à Kordongo, connaît toutes les artisanes qui vont chercher $l^{\prime}$ argile à l' endroit d' extraction. Elle connaissait certaines artisanes avant d' entamer l' apprentissage de la poterie, mais elle en a rencontré d' autres en allant chercher l' argile avec sa maman. Par exemple, elle connaît des artisanes à Merina parce que certaines lui sont apparentées. Elle leur rend visite et "apprend aussi avec elles». Elle apprend également avec sa voisine, Tama, qui occupe le même site de cuisson que sa maman. Ainsi, le maître d' une apprentie n' est pas la seule source de savoir. Par le biais d' actions collectives, la novice apprend des autres artisanes qui sont en mesure de l' aider ou de la corriger en cours de pratique. Elle participe à des actions communes au groupe et enregistre progressivement ce que l' on fait ici, dans le village. Au gré de sa participation, elle prend part au sein du groupe, définit son "identité technique », et tisse des relations avec des apprenties et des artisanes partageant notamment le même site de cuisson, le même site d' extraction ou le même marché. 
Lorsque la formation débute après le mariage, il s' agit $d^{\prime}$ un apprentissage «sur la route ${ }^{18}, s^{\prime}$ effectuant généralement auprès $d^{\prime}$ une artisane de la concession $d^{\prime}$ accueil, telle qu' une belle-mère, une co-épouse ou encore une belle-sœur. Dans ce cas, apprendre la poterie semble être une des clés de l' intégration progressive de la jeune mariée au sein de son nouveau foyer. Et $c^{\prime}$ est sur cette base qu' elle prend progressivement part au groupe de pratiquantes.

Le troisième cas de figure est celui où l' artisane est déjà formée avant d' arriver dans la localité de son époux. Dans ce cas, la potière arrive dans sa nouvelle localité avec un bagage de connaissances techniques et pratiques révélant parfois des différences notables avec «la manière de faire » des artisanes locales. La tendance principale est que la «nouvelle venue» adopte une nouvelle manière de procéder en fonction des pratiques en vigueur dans sa localité d' accueil. Ainsi, elle est amenée à rencontrer d' autres artisanes, à fréquenter $\mathrm{d}^{\prime}$ autres cadres de pratiques et $\mathrm{s}^{\prime}$ intègre progressivement, par le biais d' une pratique ajustée, dans une nouvelle communauté ayant un espace de pratique propre. Elle fait sa place tant comme épouse que comme nouvelle villageoise et nouvelle potière. Mais faire sa place ne se fait pas du jour au lendemain et les potières concernées optent généralement pour la discrétion, tant dans leurs actes que dans leurs paroles. Cette "timidité " est perceptible en termes de pratique. L' artisane arrivant dans une nouvelle localité - à l' occasion du mariage ou parce que elle et sa famille ont dû migrer à cause d' une sécheresse - ne reprend pas l' activité dès son installation. Une phase $d^{\prime}$ observation semble être de mise avant toute pratique. La nouvelle venue se rend chez la ou les artisanes « importantes » du village, généralement les artisanes les plus âgées ou réputées. Elle observe et se renseigne progressivement sur la pratique et la technique des artisanes de sa nouvelle localité, sur le site d' extraction ou encore sur le site de cuisson dans lequel elle peut éventuellement cuire ses récipients. Il arrive qu' une nouvelle venue fasse son propre "trou ", mais la plupart du temps elle s' ajoute à un groupe de cuisson déjà établi. Reprenons l' exemple de Barrow mentionné lors de l' analyse de l' étape de cuisson. Barrow, originaire de Tarsosa, est arrivée à Kordongo par le mariage. Elle y a appris la fabrication de poterie et a ensuite quitté Kordongo pour s' installer à Injer, un village situé à une quinzaine de kilomètres à l' Ouest. Après une dizaine d' années passées à Injer, elle est revenue à Kordongo. Lors de sa première installation à Kordongo, elle avait appris à préparer l' argile à la manière des artisanes de cette localité. Sa technique est restée inchangée, jusqu' à son retour à Kordongo, où elle l' a modifiée pour qu' elle corresponde à celle des artisanes de la localité, qui en avaient adopté une autre durant son absence. Barrow préparait la pâte avec deux argiles, de la chamotte, de la balle de mil et de la bouse de vache. Désormais, elle abandonne la balle de mil et la bouse de vache parce que, comme elle le dit, "J' ai trouvé qu' on ne mettait plus ici. » Barrow n' a pas posé de question: «On ne demande pas, $c^{\prime}$ est tout. » Lahia, également originaire de Kordongo, a mentionné une histoire similaire. Lors d' une sécheresse qui a sévi dans la région, elle et son époux se sont rendus à Dogondoutchi, afin de $s^{\prime}$ y installer temporairement. En arrivant dans ce nouvel espace de vie et de pratique, elle a tout d' abord observé les artisanes, $s^{\prime}$ est renseignée auprès d' elles pour connaître l' endroit où se procurer de $\mathrm{l}^{\prime}$ argile. Elle les a accompagnées sur le site $d^{\prime}$ extraction et a finalement adopté la manière de faire des artisanes de la localité. Lorsqu' elle a quitté Kordongo, Lahia décorait ses récipients, mais à son arrivée à Dogondoutchi, elle a vu que les poteries $\mathrm{n}$ 'étaient pas décorées. Lahia a cessé la décoration le temps de son installation à Dogondoutchi. Cette petite ville accueille un grand marché où l' on trouve 
actuellement des récipients décorés ou non. Il semble que cela ne soit pas par souci commercial que Lahia ait changé sa manière de faire, mais bien parce qu' il s' agissait de faire comme les artisanes de sa nouvelle localité, sans « faire de vague ». Dans ce cas, modifier et adapter sa pratique revient à s' intégrer. Précisons, enfin, que lorsque Lahia est revenue à Kordongo, elle a repris la décoration parce que «ici on $n^{\prime}$ avait pas cessé les décors ».

Ces trois exemples permettent de mieux saisir la manière dont fonctionne une communauté de potières : $l^{\prime}$ adaptation, $l^{\prime}$ intégration et la participation. Faire partie $\mathrm{d}^{\prime}$ un groupe nécessite une participation active ainsi qu' une prise de connaissance de ce que font les autres. La participation est un outil d' apprentissage (comme l' illustre l' exemple de Ussey), autant qu' un outil social permettant de tisser des relations, de s' intégrer et d' être reconnue comme faisant partie d' une même sphère d' activités et $d^{\prime}$ un même groupe de pratiquantes. La communauté de pratique fonctionne parce que les membres qui la composent sont engagés dans des actions communes, $s^{\prime}$ entraident et travaillent ensemble. Si la participation commune est génératrice $d^{\prime}$ un sentiment $d$ ' appartenance à une communauté, alors cette dernière est un élément fondamental pour comprendre l' homogénéité des pratiques au sein d' une même localité. Participer dans des cadres précis entraîne une confrontation avec différentes manières de procéder et pousse à réévaluer son travail par rapport à celui des autres. Enfin, faire partie $d^{\prime}$ un groupe, $c^{\prime}$ est aussi intégrer dans sa pratique une identité technique concordant avec celle des autres membres. Dans ce cas, une pratique "identique " légitime et matérialise l' appartenance au groupe.

\section{Niveau extravillageois : distribution des techniques au sein de la zone d' étude et effet de cadres de pratiques extravillageois}

À ce stade, il est clair que l' homogénéité des pratiques au niveau villageois est le fruit $d^{\prime}$ un fonctionnement en communauté. Néanmoins, nous ne pouvons nous limiter à ce niveau $d^{\prime}$ analyse puisque, comme nous $l^{\prime}$ avons mentionné à plusieurs reprises, les cadres et les actions collectives sont partagés par des artisanes souvent issues de différentes localités villageoises. Certaines localités sont connectées au travers d' un espace de pratiques partagé (marché ou site d' extraction) et il nous faut voir à présent si les pratiques collectives menées hors du cadre villageois ont un effet sur les «traditions techniques » à une échelle qui dépasse celle de la localité villageoise. Dans ce but, nous comparerons ici la distribution des techniques observées dans différentes communautés à deux niveaux de la chaîne opératoire - la préparation de la pâte et la décoration des récipients - et nous mettrons en parallèle les cadres de pratiques communs aux différentes communautés potières de la zone d' étude.

\section{Effet des cadres de pratiques sur la distribution spatiale des recettes de pâtes}

Deux éléments apparaissent d' emblée lorsqu' on examine la distribution spatiale des éléments ajoutés à l' argile en cours de préparation (fig. 2). 


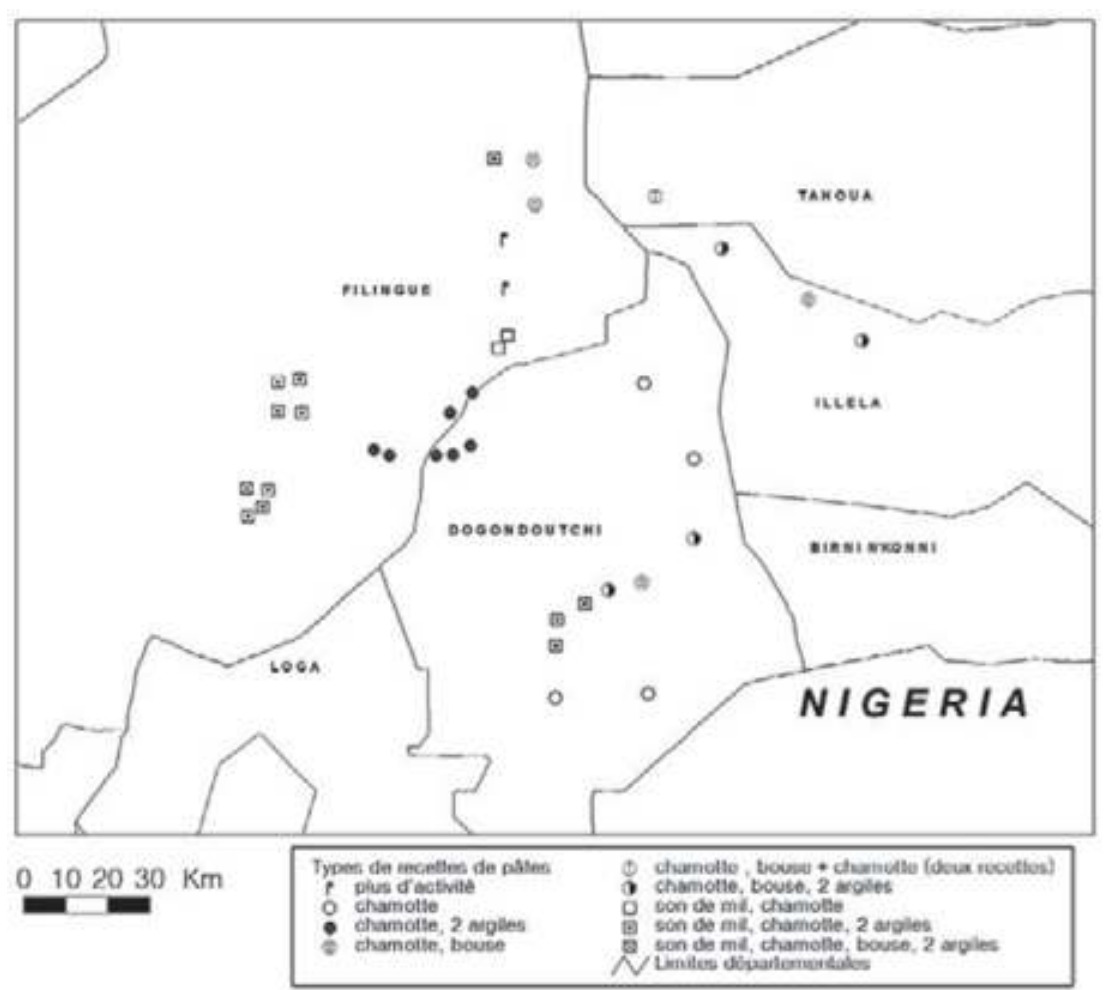

Fig. 2. - Distribution des RECETTES de PÂTES (ET LeS dÉGRAISSANTS ASSOCIÉS) UtILISÉES ACtUelLEMENT PAR LES ARTISANES

Premièrement, bien qu' il existe une grande diversité de recettes de pâtes, les potières d' une même localité utilisent généralement une recette identique pour préparer l' argile, alors que celle-ci peut considérablement varier d' une localité à l' autre. Cet élément va de pair avec le fait qu' il n' y a généralement qu' un seul site et qu' un seul marché associé à un village, toutes les artisanes $d$ ' une même localité fréquentant le même marché et le même site d' extraction (fig. 6 et 7).

Deuxièmement, l' homogénéité des techniques, flagrante au niveau local, s' observe également à une échelle micro-régionale : à l' ouest et au centre de la zone d' étude, plusieurs villages semblent former des "îlots» techniques dont les limites ne se justifient pas uniquement par la distance entre les localités (fig. 2). En observant la fréquentation des cadres de pratiques (fig. 6 et 7), on constate qu' une source d' extraction et un marché peuvent être Ícommuns à plusieurs villages. Les figures 6 et 7 mettent en évidence des ensembles de villages dont les potières fréquentent le même site $d^{\prime}$ extraction et/ou le même marché. Lorsque l' on compare la distribution des recettes de pâtes avec ces réseaux de fréquentation des sites $d$ ' extraction et des marchés, on constate une superposition presque parfaite avec les îlots techniques observés à l' ouest et au centre de la zone d' étude.

À $l^{\prime}$ est, les recettes de pâtes sont beaucoup plus diversifiées et l' homoĺgénéité technique, au-delà de l' entité villageoise, est quasiment absente. En confrontant la carte des recettes de pâtes (fig. 2) avec celle des cadres de pratiques (fig. 6 et 7), on observe que les « limites » relatives à la fréquentation des cadres de pratiques sont, en partie, analogues à celles des distributions des recettes de pâtes. Ainsi, là où les artisanes ne semblent pas partager de cadres de pratiques communs, on observe un 
accroissement de la diversité et de l' hétérogénéité des recettes de pâtes, d' un village à l' autre.

Enfin, lorsqu' on confronte la carte des recettes de pâtes actuelles à celle des pratiques plus anciennes (fig. 3), constituées sur base du témoignage des potières, on remarque qu' une série de changements ont eu lieu, qui correspondent souvent au passage d' une génération de potières à l' autre. En d' autres termes, lorsque les " maîtres » cessent l' activité, les jeunes potières confirmées prennent le relais et tendent à modifier la technique des anciennes. "Chaque génération fait ce qu' elle veut », «il faut s' adapter à son époque ", disent les potières de cette nouvelle génération pour justifier les différences avec la manière de faire de leurs aînées ${ }^{19}$. Néanmoins, on observe que les changements techniques sont adoptés par des ensembles villageois qui pérennisent les configurations techniques de la zone d' étude (fig. 2 et 3 ) et qui se transmettent les cadres de pratiques (fig. 6 et 7) d' une génération à l' autre.

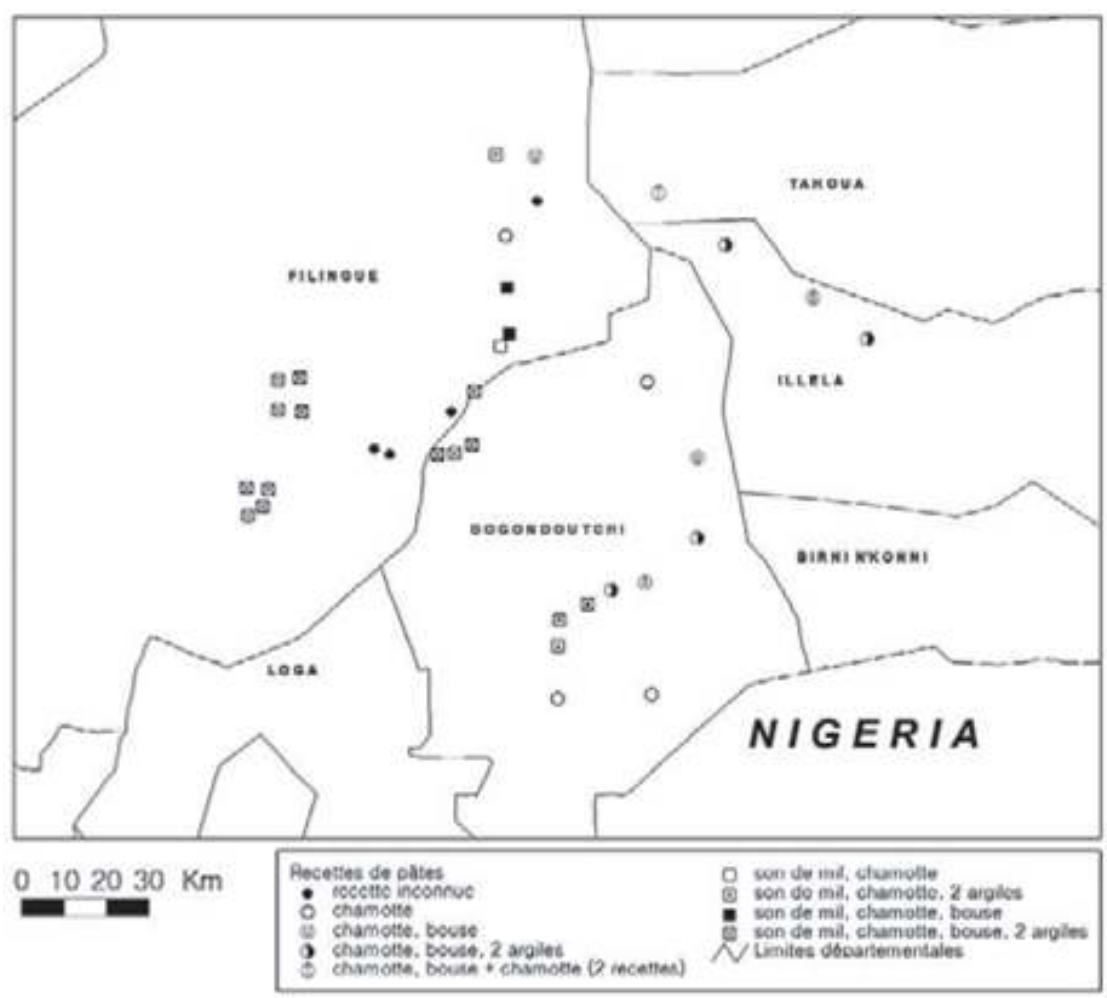

FIg. 3. - Distribution dES RECETTES DE PÂTES (ET LES DÉGRAISSANTS ASSOCIÉS) UTILISÉES PAR LES ARTISANES DE LA GÉNÉRATION PRÉCÉDENTE

\section{Effet des cadres de pratiques sur la distribution spatiale des décors}

En ce qui concerne les décors (fig. 4 et 5), j' ai choisi de m' en tenir à deux types de récipients particulièrement ubiquistes dans la production céramique de la zone $d$ ' étude : le karihi, jarre à eau placée à l' intérieur de la maison, et le tukunia, utilisé pour puiser et transporter l' eau du puits. Le karihi se distingue du tukunia par une taille plus importante et un col plus haut. Les deux récipients sont couverts $d^{\prime}$ un engobe de couleur rouge appliqué avant la cuisson.

Le premier fait frappant concernant ces récipients est la quasi-disparition de leur décor dans la zone d' étude. Cette nouvelle tendance serait apparue dans le sud-est de la zone d' étude et se serait propagée, progressivement, vers le centre. La carte des anciennes 
pratiques ornementales (fig. 5) montre l' utilisation d' impressions à la cordelette et à l' épi taillé de Blepharis ciliaris, ainsi que l' application d' un cordon d' argile au niveau du col (ill. 1 et 2). Aujourd' hui, la décoration par impression (fig. 4) a disparu du centre et du sud-est de notre zone d' étude, mais on la retrouve encore au nord, ainsi qu' à l' ouest de façon sporadique (ill. 2 et 3). L' application $d^{\prime}$ un cordon $d^{\prime}$ argile, plus fréquente que $\mathrm{l}^{\prime}$ impression à la cordelette, disparaît également dans les zones où $\mathrm{l}^{\prime}$ impression n' est plus pratiquée. En ce qui concerne la peinture blanche, celle-ci n' est généralement appliquée que sur les karihis (ill. 4). Elle était associée - et le reste encore - aux jarres à eau offertes à $\mathrm{l}^{\prime}$ occasion du mariage. Toutefois, les motifs peints (généralement en forme de points ou en bandes le long de l' épaule du récipient) semblent également en perte de vitesse et ceci plus particulièrement là où ont disparu les impressions et l' application de cordon d' argile.

Les artisanes de ces régions ont totalement cessé de décorer leurs récipients depuis une dizaine d' années. Cet abandon serait dû aux plaintes de la clientèle, faites sur les marchés et relayées dans les villages, selon lesquelles les décors par impression "gardent la poussière » et donnent un aspect sale ou négligé aux récipients. Ces plaintes ont notamment été formulées sur le marché de Doubelma, Kolefou et Bagaji. Le marché de Kolefou accueille tant des artisanes locales, fréquentant également le marché de Doubelma, que des « colporteurs » venus du sud-est (fig. 6). Ces colporteurs approvisionnent également le marché de Bagaji, fréquenté par les potières des alentours. Les marchands, venus du sud, vendent la production d' artisanes de la région de Dan Kasari, hors de la zone d' étude, qui n' ont pas de contacts directs avec celles du centre. Or, il semble que l' arrêt des décors soit plus ancien dans le sud. Les colporteurs, en amenant des récipients différents des productions locales ont peut-être encouragé l' arrêt des décors, voire dans certains cas, l' arrêt de la production du karihi. Quoiqu' il en soit, l' importation de récipients étrangers confronte les acheteurs à d' autres manières de faire et, pour peu que celles-ci emportent leur préférence, retentit sur la production locale. 


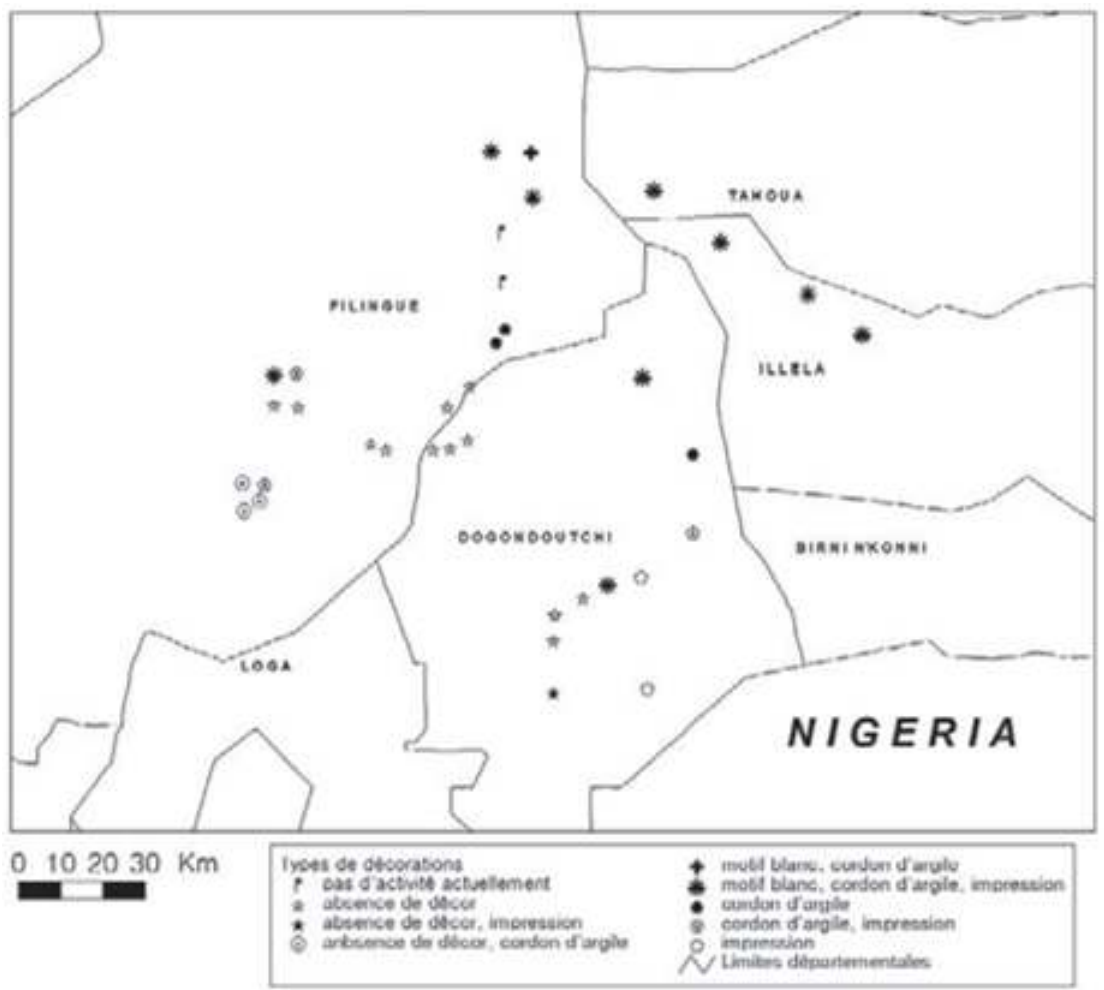

Fig. 4. - Distribution des types de déCorations actuellement RÉAliséES PAR LES ARTISANES

Cette exigence de la clientèle, liée d' une part à l' évolution des modes et, d' autre part, à l' accès à d' autres productions, semble donc avoir eu un impact important sur la production, mais également sur l' utilisation des récipients. Ainsi, le karihi était autrefois offert à la création d' un nouveau foyer. Objet utilitaire et décoratif, il faisait partie de la dot du mariage et était systématiquement décoré d' un cordon d' argile et $\mathrm{d}$ ' impressions à la cordelette. Désormais, la décoration du foyer ne se focalise plus sur cet objet, mais sur l' accumulation de bassines émaillées, empilées en colonnade le long du mur principal de la chambre (ill. 5). 


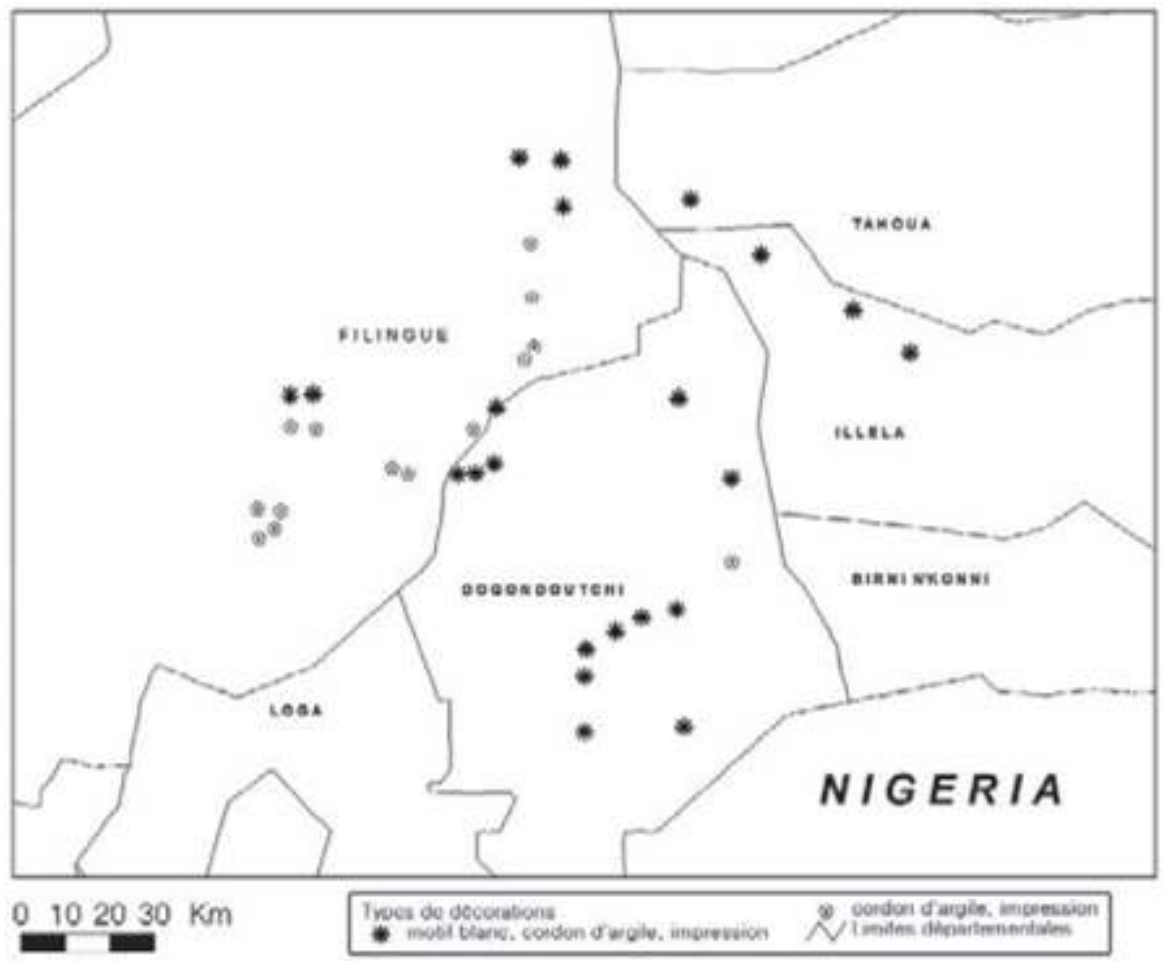

FIG. 5. - DISTRIBUTION DES TYPES DE DÉCORATIONS AUTREFOIS RÉALISÉES PAR LES ARTISANES DE LA GÉNÉRATION PRÉCÉDENTE

Le karihi semble progressivement passer de mode, remplacé par un objet d' importation plus prestigieux. Les bassines sont plus coûteuses et leur accumulation témoigne du bien-être matériel des familles. L' objet karihi n' étant plus adéquat à cet égard, la fonction de récipient à eau au sein de la maison est récupérée par le tukunia, dont la taille a été accrue. Ce phénomène est plus répandu au sud-est de la zone où les artisanes ont totalement cessé la fabrication de karihi pour se concentrer sur celle du tukunia ${ }^{20}$. De plus, certaines potières ont abandonné l' application de cordons $\mathrm{d}$ ' argile au moment où elles ont stoppé la fabrication du karihi. Elles conçoivent en effet le cordon placé à la base du col comme un renfort : sans col, le cordon devient inutile. 


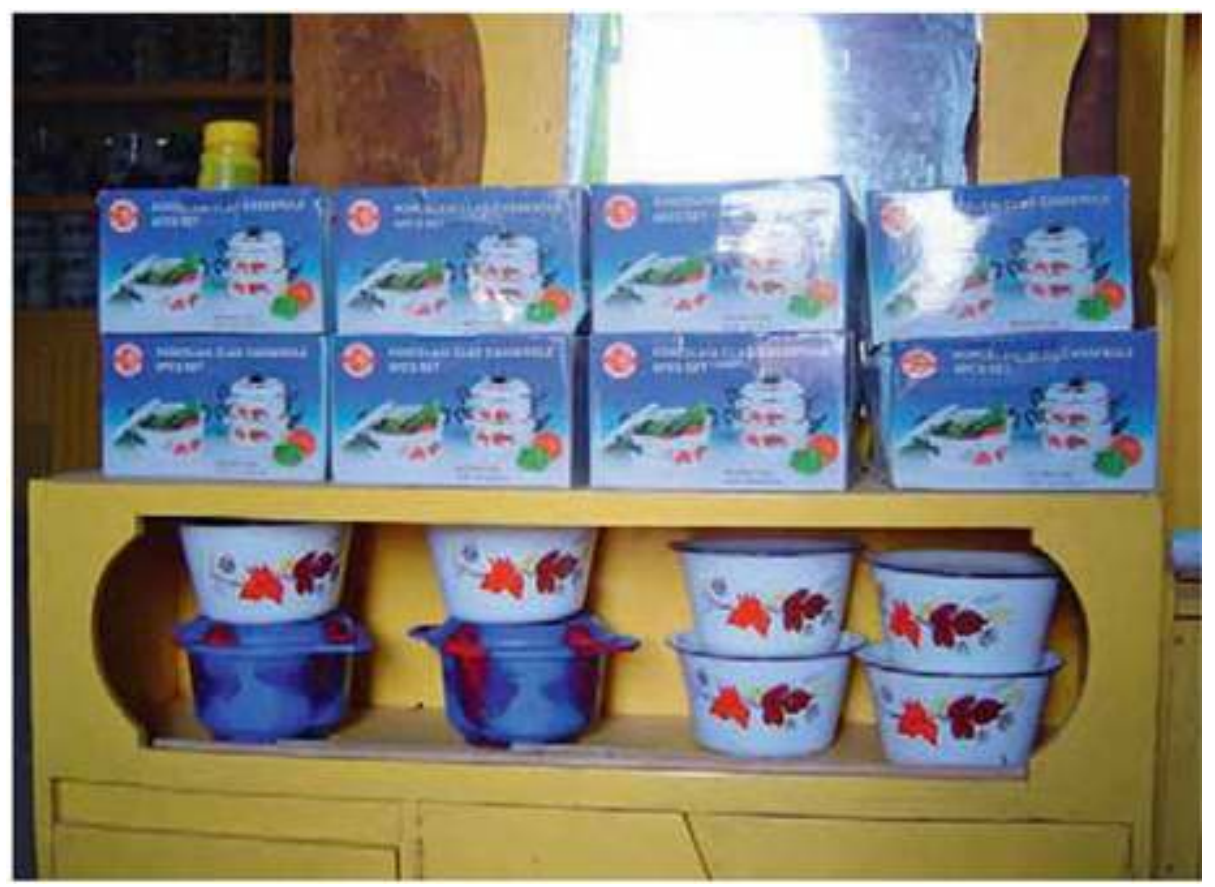

ILL. 5. - TASSES EN ÉMAIL

41 Dans le centre et à l' ouest, on constate également une diminution des décorations, mais le karihi fait toujours partie de la production principale. Alors que toutes les artisanes concernées ont stoppé la décoration des récipients, l' arrêt de la fabrication du karihi ne s' est pas généralisé. Ceci montre que les informations sont soumises à l' évaluation et jaugées en fonction de leur pertinence et de leur adéquation avec les pratiques locales.

42 En résumé, la distribution actuelle des décors présente trois zones correspondant approximativement à celles indiquées pour les recettes de pâtes. Comme pour les recettes de pâtes, l' ouest et le centre de la zone d' étude présentent de petites concentrations de villages où les artisanes utilisent la même technique et l' ont modifiée de manière identique. Enfin, les « îlots techniques » en matière de préparation de pâtes et de décorations sont à rapprocher de la fréquentation des cadres de pratiques.

43 Il ressort donc, au niveau de l' examen de la distribution spatiale des techniques qu' il peut y avoir homogénéité relative dans les pratiques, tant au niveau villageois qu' extravillageois. La lecture des cartes de distribution des recettes de pâtes et des décorations permet $d$ ' identifier les zones $d$ ' homogénéité mais pas de les expliquer. En réalité, les configurations techniques ne peuvent être comprises que si l' on prend en compte les espaces de pratiques des artisanes, $c^{\prime}$ est-à-dire le marché et le site d' extraction. Les cartes de répartition des marchés et des sites d' extraction mettent en lumière l' existence d' espaces microrégionaux au sein desquels se déroule la pratique. Les artisanes exploitant les mêmes "cadres » ont connaissance des manières de faire des autres potières qui les fréquentent. Ces cadres définissent et entretiennent l' espace dans lequel se déroule l' activité, ainsi que les rencontres qui y sont associées. Bien que des différences techniques soient notables entre l' ancienne et la nouvelle génération de potières, on constate que la fréquentation d' un site $d^{\prime}$ extraction et $d^{\prime}$ un marché ne varie quasiment jamais d' une génération à l' autre. Cet héritage d' espaces conditionne 
et maintient les relations établies entre générations d' artisanes et semble être un facteur explicatif des configurations techniques de notre zone d' étude. Ainsi, alors que la technique se modifie au fil des générations, l' évolution spatiale de ces configurations ne semble pas être aussi mouvante. Le site d' extraction et le marché font donc partie du « lot d' héritage » de l' apprentissage et/ou de l' appartenance à une communauté.
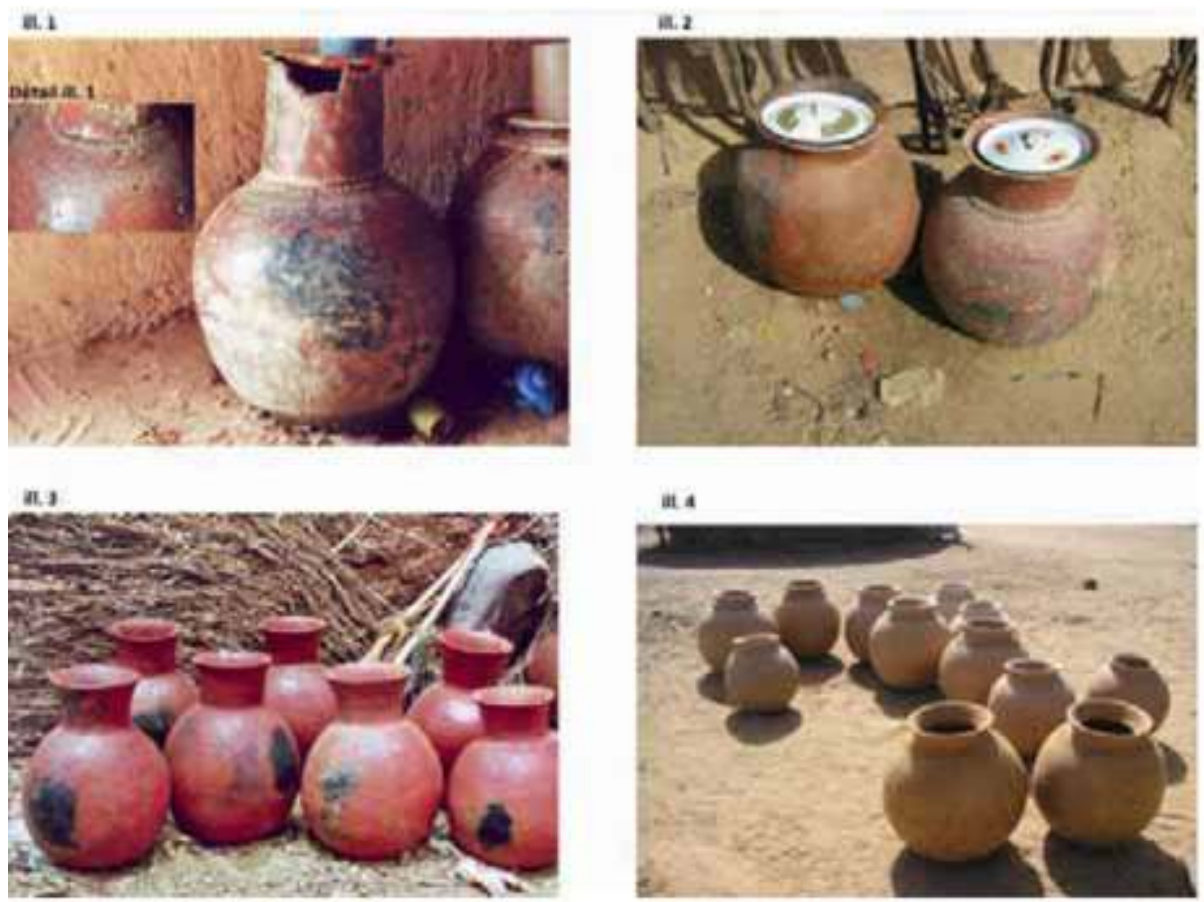

ILL. 1, 2, 3, 4. - KARIHI ET TUKUNIA

ill. 1 : Karihi décoré par impression à la cordelette et par l' application d' un cordon d' argile le long de l' épaule du récipient (récipient de la localité de Goriba).

ill. 2 : À gauche de l' image, karihi récent, non décoré. À droite de l' image, tukunia décoré par impression à la cordelette et par l' application d' un cordon d' argile le long de l' épaule du récipient (récipients de la localité de Dan kasari).

ill. 3 : Karihi sans décoration (récipients de la localité de Kordongo).

ill. 4 : Tukunia sans décoration (récipients de la localité de Dan Kasari).

Enfin, l' exemple de l' arrêt des décorations dans le centre de la zone d' étude illustre l' application d' une information, recueillie lors d' une pratique extralocale (la vente sur le marché), dans la production des artisanes concernées. Les sphères locales et extralocales sont intrinsè quement imbriquées et une série $\mathrm{d}$ ' acteurs doivent être pris en compte pour comprendre le ou les changements assimilé(s) au fil du temps. On comprend également qu' il existe une dynamique extravillageoise, à l' échelle microrégionale, qui peut être rapprochée de celle que l' on retrouve au niveau villageois en termes de partage de connaissances. Si l' homogénéité technique au niveau villageois est issue du fonctionnement en communauté de pratiques, l' homogénéité technique relative à l' échelle microrégionale est, quant à elle, liée au partage de cadres de pratiques qui fondent une conscience commune $\mathrm{d}^{\prime}$ appartenance à un même espace régional et de partage d' un même répertoire technique. 


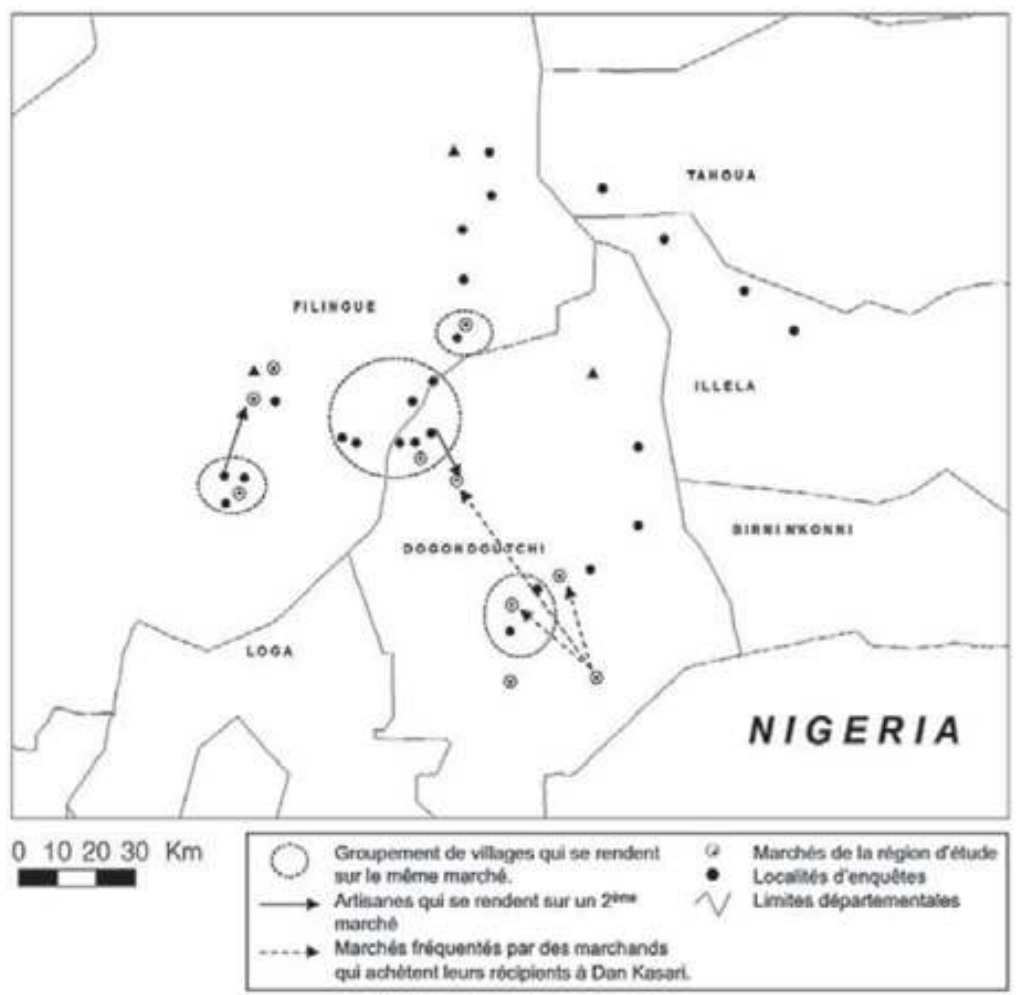

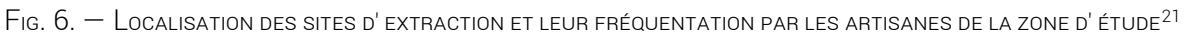

Les disparités, les changements, les continuités techniques font souvent l' objet d' explication sans réelle compréhension des mécanismes qui les animent. Autrement dit, on cherche le pourquoi sans trop se préoccuper du comment. Comme l' a signalé François Sigaut (1999: 526), «Il n' y a pas, il ne peut y avoir de transferts des techniques, car on ne peut transporter les habiletés séparément des hommes qui les possèdent et des groupes sociaux qui sont nécessaires à leur (re)production ». Si nous voulons comprendre la dynamique des "traditions techniques ", il nous faut garder à l' esprit que la potière $s^{\prime}$ inscrit dans un contexte pratique qui se définit à deux niveaux : villageois et extra-villageois. Pour être plus précis, la pratique de l' activité se déroule tant au sein de la concession, que sur un site de cuisson, un marché ou un site d' extraction. Les études qui se sont intéressées au fonctionnement d' une communauté de praticiens (Geslin \& Salembier 2002 ; Bowser \& Paton 2008), se sont effectuées dans le cadre villageois ou au sein d' une seule et même communauté, sans tenir compte de la configuration dans laquelle celle-ci était englobée. $S^{\prime}$ attacher à comprendre les situations collectives de co-construction des savoirs et savoir-faire (Geslin \& Salembier $2002: 246)$ au sein d' une communauté de pratiques n' est qu' un aspect spécifique de $\mathrm{l}^{\prime}$ étude $d$ ' une collectivité informelle. Saisir comment se construisent et évoluent les savoirs au sein $d^{\prime}$ un groupe nécessite de situer la communauté dans son contexte global de pratiques et d' établir les connexions éventuelles avec les membres d' autres communautés. Ainsi, alors qu' il est reconnu que les artisanes sont engagées dans une dynamique de groupe au niveau villageois, notre étude tend à montrer qu' une pareille dynamique est présente à une échelle plus large. Même lorsque l' artisane est isolée au sein de son village, elle s' ancre dans un espace particulier et tisse des relations - ou tout au moins entre en interaction - avec $d^{\prime}$ autres praticiennes par le biais $d^{\prime}$ 
opérations collectives hors du contexte villageois. Négliger les cadres de pratiques et les interactions s' y déroulant, $c^{\prime}$ est en quelque sorte nier le contexte et l' histoire " quotidienne » qui ont façonné et façonnent les « traditions techniques ». Partant de l' homogénéité flagrante au niveau villageois et apparente au sein de zones microrégionales, la coopération et la constance des cadres de pratiques semblent être deux facteurs explicatifs des configurations techniques en présence dans notre région d' étude.

Notre ambition est à présent de mieux comprendre l' articulation entre la circulation de connaissances techniques (entre les potières $d^{\prime}$ un même village et $d^{\prime}$ autres localités) et les divers cadres de pratiques identifiés dans cette première étude. Il nous faudra caractériser la nature et le degré d' interaction au sein de chaque cadre, afin d' appréhender les mécaniques qui orientent les choix posés par les artisanes d' une même localité.

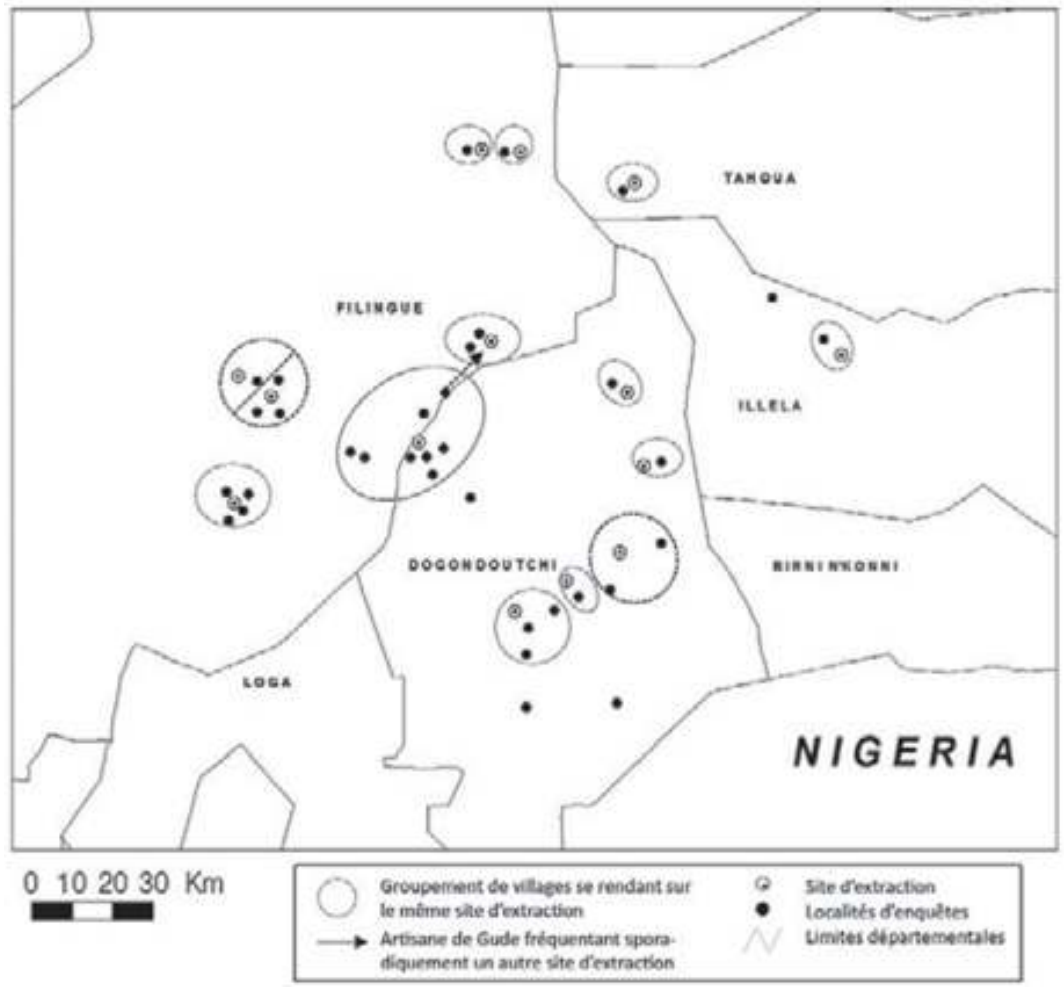

Fig. 7. - LocAlisation des MARChÉS ET LEUR FRÉQUENTATION PAR LES ARTISANES DE LA ZONE D' ÉTUDE ${ }^{22}$

Une série d' éléments doivent donc être pris en compte si l' on veut comprendre comment s' accrochent le social et le technique. Premièrement, il nous faut cerner l' espace de pratiques quotidien des artisanes et les interactions qui y ont cours, afin de comprendre les configurations techniques de la zone d' étude. Deuxièmement, si nous voulons situer et caractériser les interactions entre les artisanes, il nous faut établir la nature des relations entretenues sur les deux niveaux $d$ ' analyses (villageois et extravillageois) et leur impact sur la continuité ou les changements techniques. Troisièmement, le degré de participation implique un statut (apprentie, potière confirmée, maître, nouvelle venue, ancienne) particulier qui évolue en fonction de la pratique et définit la place qu' occupe une artisane au sein de sa communauté. Il est donc nécessaire d' établir les divers degrés de participation et $d$ ' identifier les artisanes ayant le plus d' influence au sein du groupe de fabricantes. Enfin, il nous faut prendre 
en compte le renouvellement successif de générations $d^{\prime}$ artisanes, puisque cette dynamique générationnelle est susceptible $d$ ' amener des modifications dans la pratique.

\section{BIBLIOGRAPHIE}

ARNold, D. E. 1972 ” Native Pottery Making in Quinua, Peru », Anthropos, 67 : 858-872.

BERNS, M. C. 1989 " Ceramic Clues : Art History in the Congola Valley ", African Arts, 22 (2) : 48-59.

BOWSER, B. J. \& PATON, J. Q. 2008 " Learning Strategies and Cultural Transmission of Pottery Style : Women's Life Histories and Communities of Practice in the Ecuadorian Amazon ", in M. T. STARK, B. J. BOWSER \& L. HORNE (eds.), Cultural Transmission and Material Culture Breaking Down Boundaries, Tucson, University of Arizona Press : 105-129.

BRown, J. S. 1991 " Organizational Learning and Communities of Practice : Toward a Unifying View of Working, Learning and Innovation », in M. D. COHEN \& L. S. SPRoulL (eds.), Organizational Learning, London, ENG : 59-82.

BRown, J. S., Collins, A. \& Duguid, P. 1989 " Situated Cognition and the Culture of Learning ", Education Research, 18 (1) : 32-42.

CHANAL, V. 2000 " Communautés de pratique et management par projet : à propos de l' ouvrage de Wenger (1998), Communities of Practice. Learning, Meaning, and Identity ", M@n@gement, 3 (1) : 1-30. <//www.management-aims.com/PapersMgmt/31Chanal.html>.

CReSSWeLl, R. 1996 Prométhée ou Pandore ? Propos de technologie culturelle, Paris, Kimé.

2002 " Geste technique et fait social. La technique est-elle dans le social ou face à lui ? ", Techniques et Culture, <http://tc.revues.org/ 1576>.

GESLIN, P. \& SALEMBIER, P. 2002 " La fleur, l' épice : usage social du corps et communauté de pratique dans la relance de produits authentiques ", Revue d' anthropologie des connaissances, 16 (2) : 244-259.

Gosselain, O. P. 2000 " Materializing Identities : An African Perspective ", Journal of Archaeological Method and Theory, 7 (3) : 187-217.

Gosselain, O. P. \& LivingSTONE-SMith, A. 2005 " The Source. Clay Selection and Processing Practices in Sub-Saharan Africa ", in A. Livingstone-SMith, D. Bosquet \& R. MARTINEAu (eds.), Pottery Manufacturing Processes : Reconstitution and Interpretation, Oxford, BAR International Series, 1359 : 33-47.

Guille-ESCURET, G. 2003 " Efficacité technique, efficacité sociale. Le technique est-il dans le social ou face à lui ?", Techniques et Culture, <http://tc.revues.org/ 1414>.

HAMANI, D. 1993 " Proto-hausa et haousa », Vallées du Niger, Paris, Éditions de la réunion des musées nationaux : 192-203.

DE LATOUR, É. 1984 " Maîtres de la terre, maîtres de la guerre ", Cahiers d' Études africaines, XXIV (3), $95: 273-297$. 
LAVE, J. 1988 Cognition in Practice, New York, Cambridge University Press.

LEMONNIER, P. 1993 Technological Choices. Transformation in Material Culture Since Neolithic, London, Routledge.

LEROI GOURHAN, A. 1964 Le Geste et la Parole. Technique et Langage, Paris, Albin Michel.

LIVINGSTONE-SMITH, A. 2001 Chaînes opératoires de la poterie. Références ethnographiques, analyse et reconstitution, Thèse de doctorat, Bruxelles, Faculté de Philosophie et Lettres, Université Libre de Bruxelles.

MAHIAS, M.-C. 2002 Le barratage du monde. Essais d' une anthropologie des techniques en Inde, Paris, Éditions de la Maison des sciences de l' homme.

NicolAS, G. 1975 " Les catégories d' ethnie et de fraction ethnique au sein du système social hausa ", Cahiers d' Études africaines, XV (3), 59 : 399-441.

SÉRÉ DE RIVIÈRES, E. 1965 Histoire du Niger, Paris, Berger-Levrault.

SIGAUT, F. 1999 Dans le sillage des techniques. Hommage à Robert Cresswell, Paris, L' Harmattan.

STARK, M. T. 1999 " Social Dimensions of Technical Choice in Kalinga Ceramic Traditions ", in E. S. CHILTON (ed.), Material Meanings : Critical Approaches to the Interpretation of Material Culture, Salt Lake City, University of Utah Press : 24-43.

Suchman, L. A. 1987 Plans and Situated Action: Problem of Human Machine Interaction, Cambridge, Eds. Roy Pea \& John Seely Brown, Cambridge University Press.

VisetTI, Y.-M. 1989 Compte-rendu : « Lucy A. Suchman, Plans and Situated Action. The Problem of Human Machine Communication », Intellectita, $7: 67-96$.

WENGER, É. \& LAVE, J. 1991 Situated Learning : Legitimate Peripheral Participation, Cambridge, Cambridge University Press.

WENGER, É. 1998 Communities of Practice. Learning, Meaning, and Identity, Cambridge, Cambridge University Press.

\section{NOTES}

1. Nous entendons par cadres de pratiques les lieux dans lesquels se déroulent l' une ou l' autre étape de la chaîne opératoire. Ces lieux sont investis d' actions spécifiques, collectives ou individuelles.

2. Les missions réalisées en 2004, 2005, 2006 et 2007 ont débuté dans le cadre du projet $\mathrm{d}^{\prime}$ ' «Atlas des traditions céramiques du Niger » dirigé par Olivier Gosselain, et se sont poursuivies suite à l' octroi d' une bourse de recherche Mini-Arc de l' Université libre de Bruxelles (ULB). Je tiens à remercier Dulla Sindy, ancien chauffeur de l' IRSH (Institut de recherches en sciences humaines) de Niamey, pour sa précieuse collaboration scientifique et humaine en tant que guide et interprète lors des enquêtes. Je remercie également le directeur (Abdoulaï Maga) et son équipe de l' IRSH de Niamey. Enfin, mis à l' honneur également le Centre d' anthropologie culturelle et le Laboratoire de géographie humaine (Faculté des sciences), et en particulier Eléonore Wolff, de l' ULB.

3. Langue parlée par plus de la moitié des Nigériens. 
4. Ils seraient venus de Daura, conduits par une reine prêtresse, la Sarraounyia (DE LATOUR $1984: 276$ ). $C^{\prime}$ est cette première couche de population, enrichie au fil du temps de petits groupes qui auraient adoptés leurs pratiques, que l' on appelle Azna/Anne qui sont les maitres de la terre (NICOLAS 1975).

5. Le terme Arewa fait référence à la dynastie arauci (adjectif correspondant au nom propre Arewa) fondée par Akazama, né de l' union de la fille de Bawra et du prince qui fut à la tête des guerriers venus du Bornu au XVI e siècle (DE LATOUR 1984 : 276).

6. Nous utiliserons, tout au long de cette étude, les termes de potière et $d^{\prime}$ artisane puisque la pratique potière est exclusivement féminine dans cette partie du Niger.

7. L' activité potière se déroule pendant la saison sèche, approximativement de février à mai. En dehors de cette période, les artisanes sont occupées aux tâches agricoles et font parfois $\mathrm{du}$ " petit commerce » (beignets, arachides, etc.).

8. La potière s' installe à l' extérieur dans un endroit généralement prévu à cet effet, à l' abri du soleil.

9. Les deux argiles - souvent extraites comme des blocs de terre - sont placées dans l' eau pour les rendre plus malléables. Lorsque celles-ci sont humidifiées, l' artisane prend l' argile blanche et $\mathrm{y}$ ajoute, généralement, une quantité inférieure d' argile noire. Avec ces deux argiles elle forme une motte qu' elle place sur une natte. Elle la malaxe et y ajoute un ou plusieurs dégraissants. Les différents matériaux ajoutés à $\mathrm{l}^{\prime}$ argile sont la chamotte (tesson de céramique pilé), le crottin de vache et la balle de mil. Comme nous le verrons par la suite, leur combinaison est variable et se modifie au cours du temps. Une fois les dégraissants ajoutés à l' argile, la potière malaxe la pâte jusqu' à ce qu' elle juge sa consistance et sa texture conformes à ses exigences personnelles.

10. Dans notre zone d' étude, la technique de façonnage utilisée est le moulage sur forme convexe.

11. Nous avons assisté, à plusieurs reprises, à des démonstrations d' artisanes qui reconnaissent, par le toucher notamment, les récipients de leur collègue, ainsi que des apprenties identifiant la production de leur maitre.

12. La dépression, circulaire, mesure 3 à $4 \mathrm{~m}$ de large et de 20 à $30 \mathrm{~cm}$ de profondeur. Après avoir tapissé le fond de tiges de mil, les potières placent les pots en cercle en les couchant sur le flanc. Elles les couvrent d' une succession de couches de combustibles tels que des tiges de mil, des excréments d' animaux (vache ou âne), du son de mil ou encore de la paille.

13. $L^{\prime}$ autre canal de distribution est la commande, passée généralement par les habitants du village.

14. Il nous faut préciser que seul le point de cuisson est propre aux artisans d' un même village. L' endroit d' extraction et le marché mettent en contact des artisans de villages différents. Ce dernier point est crucial pour notre recherche et sera évoqué par la suite.

15. La notion de communauté de pratique est mentionnée pour la première fois au début des années 1990 dans l' ouvrage commun d' Étienne WENGER et Jean LAVE (1991).

16. Par exemple, lors de l' extraction ou de la cuisson des récipients, la potière est souvent accompagnée ou aidée par les enfants de sa concession ou par son/ses apprentie(s), enfants ou adultes. 
17. Comme l' ont montré WENGER et LAVE (1991), l' apprentissage informel d' une pratique professionnelle se construit par la participation d' un acteur à la pratique collective et dessine une trajectoire allant de la périphérie vers le centre.

18. Comme le disent les potières de Merina.

19. Cet élément, qui ne sera pas approfondi ici, apparaît comme déterminant pour appréhender les changements dans les «traditions techniques » sur des périodes de temps relativement courtes.

20. Utilisé tant pour aller chercher l' eau que comme jarre à eau au sein de la maison.

21. Certaines localités ne sont pas associées à un site d' extraction puisque ce ou ces derniers nous sont, pour l' instant, inconnus.

22. Certaines localités ne sont pas associées à un marché puisque ce ou ces derniers nous sont, pour l' instant, inconnus.

\section{RÉSUMÉS}

RÉSUMÉ

À première vue, la poterie est une activité qui se pratique de manière individuelle: chaque potière maîtrise son ouvrage du début à la fin de la chaîne opératoire ; chaque artisane possède un espace réservé au sein de la concession; le profit financier de la production est propre, puisque la totalité des gains, lors de la vente des récipients, revient à l' artisane. Néanmoins, les enquêtes de terrain menées dans la région de l' Arewa (centre-sud du Niger) révèlent qu' à chaque étape de la chaîne opératoire, $l^{\prime}$ artisane est en contact plus ou moins étroit avec d' autres praticiennes: apprenties, artisanes de sa localité ou d' autres localités. Si ces contacts sont parfois informels, des regroupements ont lieu régulièrement par le biais de certaines étapes de la chaîne opératoire tels que la cuisson, le site d' extraction et le marché. Ces étapes s' effectuent au sein de quatre cadres de pratique : l' atelier et le lieu de cuisson (à l' échelle villageoise); le site d' extraction et le marché (à l' échelle microvillageoise). L' ensemble de ces cadres constitue l' espace de pratique des artisanes au sein duquel se déroulent des actions collectives, des échanges de connaissances et une collaboration entre les potières. Ce papier propose d' une part, de mettre en évidence les points de contacts qui lient et interconnectent les potières d' une même localité et des différentes localités de la zone d' étude et, $d$ ' autre part, d' évaluer l' impact de l' espace partagé de pratique sur la distribution des techniques céramiques. Nous tenterons, également, d' évaluer l' étendue spatiale des connaissances techniques des potières afin d' aborder comment, par quelle voie et sous quelle forme circulent les connaissances techniques.

\section{ABSTRACT}

Frameworks of Practice and Circulation of Knowledge between the Potters in Arewa (Niger).

Pottery is an activity supposedly practiced alone: the potter possesses her own workshop in the village, makes her own pots and the sales are totally for her. Nevertheless, the fields enquiries led in the area of Arewa (central-southern Niger) reveal that at each stage of the "operating chain" (or "chaine opératoire"), the craftswoman is in more or less close contact with others practitioners (as apprentices; craftswomen from her locality and from others localities). Whether these contacts are organized or informal, the gatherings take usually place in the 
context of some operating chain' s stage situated at different scales: village scale (as the cooking site) and microregional scale (as the clay source and the market). The practice space' s sharing generates collective actions, knowledge exchanges and craftswoman's collaboration. When a potter makes a pot, she is not isolated but inscribes her practice in a known and lived world. Her technique is as much marked by her apprenticeship than by her familial, linguistic and villager identity as well as her interactions with others practitioners from her village or from elsewhere. If we admit that every practice is situated and that the situation gives meaning to practice, it becomes imperative to examine the situations of practice as far as the frameworks in which these situations take place. This paper proposes a study of the contacts' points and degrees which link and interconnect the potters from different localities of this area, and the impact of the shared space of practice on the technical ceramics' distribution. Analysing the "context of practice", we would explain the technical configurations of this area.

INDEX

Mots-clés : Niger, cadres de pratique, céramique, chaîne opératoire, ethno-archéologie

\section{AUTEUR}

\section{CLAIRE CORNIQUET}

CReA-Patrimoine, Université Libre de Bruxelles, Belgique. 